\title{
Comparing Laser Ray Tracing, the Spatially Resolved Refractometer, and the Hartmann- Shack Sensor to Measure the Ocular Wave Aberration
}

\author{
ESTHER MORENO-BARRIUSO, PhD, SUSANA MARCOS, PhD, RAFAEL NAVARRO, PhD, \\ and STEPHEN A. BURNS, PhD
}

Instituto de Optica "Daza de Valdés", Consejo Superior de Investigaciones Cientificas (C.S.I.C.), Serrano 121, 28006 Madrid, Spain (EM$B, S M, R N)$ and Schepens Eye Research Institute, Boston Massachusetts (SAB)

\begin{abstract}
Purpose. To compare quantitatively three techniques to measure the optical aberrations of the human eye: laser ray tracing (LRT), the Hartmann-Shack wavefront sensor (H-S), and the spatially resolved refractometer (SRR). LRT and H-S are objective imaging techniques, whereas SRR is psychophysical. Methods. Wave aberrations were measured in two normal subjects with all three techniques implemented in two different laboratories. Results. We compared the experimental variability of the results obtained with each technique with the overall variability across the three methods. For the two subjects measured (RMS wavefront error $0.5 \mu \mathrm{m}$ and $0.9 \mu \mathrm{m}$, respectively), we found a close agreement; the average standard deviation of the Zernike coefficients within a given method was $0.07 \mu \mathrm{m}$, whereas the average global standard deviation across techniques was $0.09 \mu \mathrm{m}$, which is only slightly higher. Conclusions. There is a close match between the Zernike coefficients obtained by LRT, H-S, and SRR. Thus, all three techniques provide similar information concerning wave aberration when applied to normal human eyes. However, the methods are operationally different, and each has advantages and disadvantages depending on the particular application. (Optom Vis Sci $2001 ; 78: 152-156)$
\end{abstract}

Key Words: ocular aberrations, laser ray tracing, Hartmann-Shack, spatially resolved refractometer, aberrometer

$\mathrm{n}$ recent years, there has been a renewed effort to develop techniques for estimating the wave aberration of the human eye, ${ }^{1-7}$ partly stimulated by the interest in assessing the changes in optical quality produced by refractive surgery ${ }^{8,9}$ and by the desire to compensate the ocular aberrations to achieve diffraction-limited optics. $^{10-12}$

Although a large number of techniques are now available, little work has been done so far to assess their equivalence ${ }^{13-15}$ or to establish which technique is best suited for a particular situation. In the current study, three different techniques, laser ray tracing (LRT), the spatially resolved refractometer (SRR), and the Hartmann-Shack wavefront sensor (H-S), have been compared in two subjects. Their principle and optical setups have been thoroughly described elsewhere. ${ }^{3,4,16}$ LRT and H-S are objective techniques, whereas SRR is psychophysical. They all share a common basic principle ${ }^{17}$ : the pupil is sampled at a set $\left(\xi_{i}, \eta_{i}\right)$, for $i=1, \ldots$ of locations, forming a given pattern. The raw data represent the set of ray aberrations $\left[\Delta \mathrm{x}^{\prime}\left(\xi_{\mathrm{i}}, \eta_{\mathrm{i}}\right), \Delta \mathrm{y}^{\prime}\left(\xi_{\mathrm{i}}, \eta_{\mathrm{i}}\right)\right]$ corresponding to those pupil locations $s^{\mathrm{a}}$ and are proportional to the slope, or partial derivatives, of the wave aberration $(\mathrm{W})^{18}$ :

$$
\Delta \mathrm{x}^{\prime}=\frac{1}{\mathrm{R}_{\mathrm{p}}} \frac{\partial \mathrm{W}(\bar{\xi}, \bar{\eta})}{\partial \bar{\xi}} ; \Delta \mathrm{y}^{\prime}=\frac{1}{\mathrm{R}_{\mathrm{p}}} \frac{\partial \mathrm{W}(\bar{\xi}, \bar{\eta})}{\partial \bar{\eta}}
$$

where $\bar{\xi}=\xi / R_{P}, \bar{\eta}=\eta / R_{P}$ are dimensionless canonical pupil coordinates and $\mathrm{R}_{\mathrm{p}}$ is the pupil radius. Ray aberrations are also given in dimensionless tangent units. If they are small, the tangent is equal to the angle.

The joint representation of all the raw data $\left[\Delta x^{\prime}\left(\xi_{i}, \eta_{i}\right)\right.$, $\left.\Delta \mathrm{y}^{\prime}\left(\xi_{\mathrm{i}}, \eta_{\mathrm{i}}\right)\right]$, for $\mathrm{i}=1, \ldots$. n constitutes the spot diagram, which can be taken as a rough estimate of the shape of the retinal point spread

aThe raw data correspond to the average slope of the wave aberration over this sample area. This value is associated to the pupil location of the center of the sample beam for further data fitting. This is a good approximation in normal eyes. See reference 2 (Sections $2 \mathrm{~F}$ and 3 ) for further details. 
function. ${ }^{4}$ Instead of directly integrating Equation 1 to estimate $\mathrm{W}$, it is numerically more stable to consider an expansion of $\mathrm{W}$ in terms of Zernike polynomials. Here we have considered a seventhorder approximation; that is, 35 terms $^{19}$ :

$$
\mathrm{W}(\bar{\xi}, \bar{\eta}) \alpha \sum_{\mathrm{k}=1}^{35} \mathrm{Z}_{\mathrm{k}} * \mathrm{P}_{\mathrm{k}}(\bar{\xi}, \bar{\eta})
$$

where $Z_{k}$ are the coefficients of the expansion, in microns, and $\mathrm{P}_{\mathrm{k}}$ are dimensionless Zernike polynomials.

By computing the partial derivatives of Equation 2 and substituting them in Equation 1 the wave aberration is estimated by a standard least-squares fitting of the raw data to the partial derivatives of the Zernike polynomials for each pupil location sampled. ${ }^{20}$

\section{METHODS}

The experimental approach differs between techniques. Fig. 1 shows simplified schematic diagrams of the setups. In LRT (Fig. 1a), a set of parallel laser Gaussian pencils is sequentially delivered

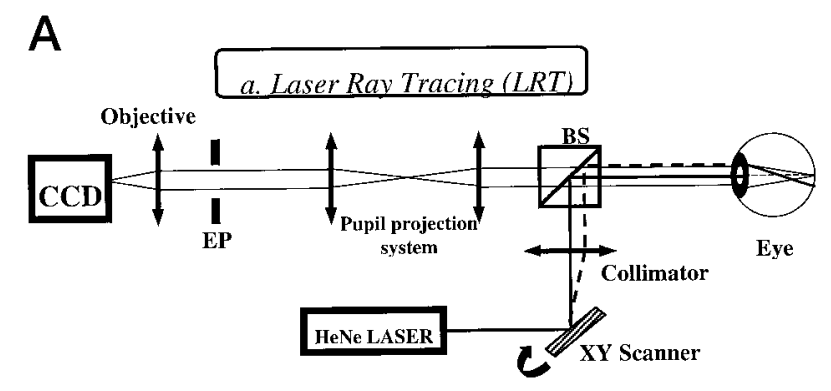

B
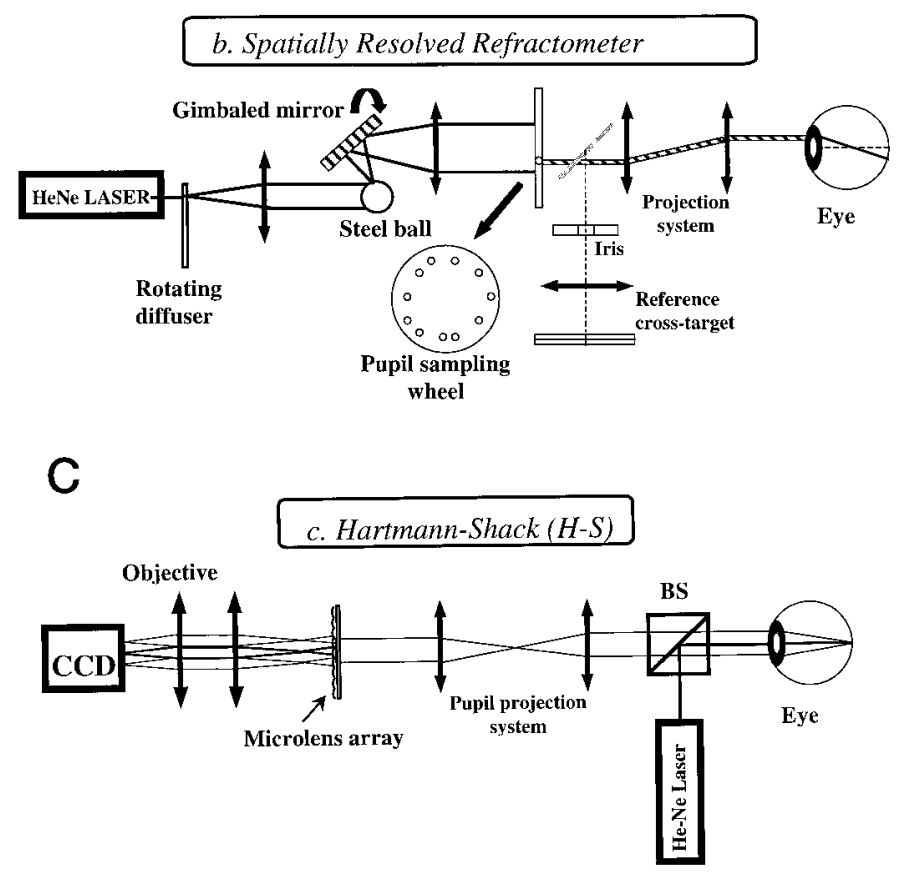

FIGURE 1.

Schematic diagrams of the basic setup for the three techniques: laser ray tracing (a), spatially resolved refractometer (b), Hartmann-Shack wavefront sensor (c). onto the eye through different pupil locations $\left(\xi_{\mathrm{i}}, \eta_{\mathrm{i}}\right)$. Each ray forms a Gaussian spot on the retina (assuming that for the size of the entry beam, the effective pupil is diffraction limited) and an aerial image of the retina (which is the cross-correlation of the retinal point spread function with the Gaussian $\operatorname{spot}^{21}$ ) is then relayed onto a CCD camera. The image is recorded, and its centroid is subsequently computed as a maximum-likelihood estimate of its position $\left(\mathrm{X}^{\prime}\left(\xi_{\mathrm{i}}, \eta_{\mathrm{i}}\right), \mathrm{Y}^{\prime}\left(\xi_{\mathrm{i}}, \eta_{\mathrm{i}}\right)\right)$. The joint plot of all the centroids constitutes the spot diagram. In the presence of aberrations, the spots (located in $\left.\left(\mathrm{X}^{\prime}, \mathrm{Y}^{\prime}\right)\right)$ are displaced from their reference positions $\left(\mathrm{X}^{\prime}=\mathrm{X}_{0}^{\prime} ; \mathrm{Y}^{\prime}=\mathrm{Y}^{\prime}{ }_{0}\right)$, and the geometrical aberration is defined by the magnitude of these displacements (given here in dimensionless tangent units):

$$
\begin{aligned}
& \Delta \mathrm{x}^{\prime}\left(\xi_{\mathrm{i}}, \eta_{\mathrm{i}}\right)=\left[\mathrm{X}^{\prime}\left(\xi_{\mathrm{i}}, \eta_{\mathrm{i}}\right)-\mathrm{X}_{0}^{\prime}\left(\xi_{\mathrm{i}}, \eta_{\mathrm{i}}\right)\right] \\
& \Delta \mathrm{y}^{\prime}\left(\xi_{\mathrm{i}}, \eta_{\mathrm{i}}\right)=\left[\mathrm{Y}^{\prime}\left(\xi_{\mathrm{i}}, \eta_{\mathrm{i}}\right)-\mathrm{Y}_{0}^{\prime}\left(\xi_{\mathrm{i}}, \eta_{\mathrm{i}}\right)\right]
\end{aligned}
$$

We use a unique reference in LRT $\left(\mathrm{X}_{0}^{\prime}, \mathrm{Y}_{0}^{\prime}\right)$, which is the location of the spot for the chief ray. In LRT, the first pass is the measurement pass, whereas in the second pass, all rays are affected equally by the overall aberrations (assuming isoplanatism).

SRR (Fig. 1b) is a psychophysical technique. As in LRT, the pupil is sampled by sequentially projecting diffraction-limited apertures (drilled in a spinning wheel) at different locations $\left(\xi_{\mathrm{i}}, \eta_{\mathrm{i}}\right)$ on the pupil. A focusing block (not shown in Fig 1b) allows for defocus compensation. Due to the aberrations, the subject will perceive the spots in different retinal locations. For each entry pupil, the task consists of having the patient tilt the incoming beam a given angle along both axes $\left(\alpha_{\mathrm{x}}\left(\xi_{\mathrm{i}}, \eta_{\mathrm{i}}\right), \alpha_{\mathrm{y}}\left(\xi_{\mathrm{i}}, \eta_{\mathrm{i}}\right)\right)$ by means of a joystick until the spot is perceived to be aligned to a reference cross, viewed through the center of the pupil (reference channel in Fig. 1b). The tilt equals the wavefront slope, but with opposite sign so that the ray aberration is cancelled out. The ray aberration $\left[\Delta \mathrm{x}^{\prime}\left(\xi_{\mathrm{i}}, \eta_{\mathrm{i}}\right)\right.$, $\left.\Delta \mathrm{y}^{\prime}\left(\xi_{\mathrm{i}}, \eta_{\mathrm{i}}\right)\right]$ is computed from Equation 3 using $\left(\mathrm{X}^{\prime}\left(\xi_{\mathrm{i}}, \eta_{\mathrm{i}}\right), \mathrm{Y}^{\prime}\left(\xi_{\mathrm{i}}\right.\right.$, $\left.\left.\eta_{\mathrm{i}}\right)\right)=\left(-\alpha_{\mathrm{x}}\left(\xi_{\mathrm{i}}, \eta_{\mathrm{i}}\right),-\alpha_{\mathrm{y}}\left(\xi_{\mathrm{i}}, \eta_{\mathrm{i}}\right)\right)$ and considering the center of the pupil as the aberration-free reference.

In the H-S wavefront sensor (Fig. 1c), a monolithic microlens array, located in a plane conjugate to the exit pupil, samples the wavefront in parallel. Each lenslet selects a portion of the wavefront of the size of the lenslet and forms a spot of light at its focal plane. A single snapshot provides a grid of image spots, which is imaged onto a CCD camera. Each spot is the cross-correlation between the point spread function of the eye (first pass) and that of a single microlens (second, measurement pass). For an aberration-free eye, the emerging wavefront is flat, and thus the grid of image spots has a spatial distribution $\left(\mathrm{X}_{0}{ }^{\prime}\left(\xi_{\mathrm{i}}, \eta_{\mathrm{i}}\right), \mathrm{Y}_{0}{ }^{\prime}\left(\xi_{\mathrm{i}}, \eta_{\mathrm{i}}\right)\right)$ identical to that of the microlenses themselves. In the presence of aberrations, the image spots $\left(\mathrm{X}^{\prime}\left(\xi_{\mathrm{i}}, \eta_{\mathrm{i}}\right), \mathrm{Y}^{\prime}\left(\xi_{\mathrm{i}}, \eta_{\mathrm{i}}\right)\right)$ are shifted from their reference position. This shift is proportional to the average slope of the wavefront across the microlens pupil (Equation 1). By applying Equation 3, subtracting the test from the reference coordinates of the centroids, we obtain the geometrical aberrations $\left[\Delta \mathrm{x}\left(\xi_{\mathrm{i}}, \eta_{\mathrm{i}}\right), \Delta \mathrm{y}\left(\xi_{\mathrm{i}}, \eta_{\mathrm{i}}\right)\right]$ and the spot diagram.

Table 1 summarizes the characteristic features of each of the three techniques, both the ones inherent to the method itself and the ones due to the particular setup used for this experiment (but that could be modified in alternative implementations). For this comparative study, two different laboratories were involved: we 
TABLE 1.

Specifications for Laser Ray Tracing, Spatially Resolved Refractometer, and Hartmann-Shack Wavefront Sensor implemented for this study

\begin{tabular}{|c|c|c|c|}
\hline & $\mathrm{LRT}^{\mathrm{a}}$ & SRR & $\mathrm{H}-\mathrm{S}$ \\
\hline Objective/psychophysical & Objective & Psychophysical & Objective \\
\hline Parallel/sequential & Sequential & Sequential & Parallel \\
\hline No. of samples taken & 37 & 37 & $91^{b}$ \\
\hline Sample diameter (mm) & $\sim 0.6$ & 1 & $0.5^{b}$ \\
\hline Sampling step (mm) & 1 & 1 & $0.6^{b}$ \\
\hline Sampling pattern (shape) & Rectangular & Rectangular & Hexagonal \\
\hline Effective pupil sampled $(\mathrm{mm})^{C}$ & 6.6 & 7.2 & 6.6 \\
\hline Measurement in first/second pass & First & First & Second \\
\hline Entry pupil size $(\mathrm{mm})$ & $\sim 0.6$ & 1 & $\sim 0.6$ \\
\hline Exit pupil size $(\mathrm{mm})$ & $3^{d}$ & $\mathrm{~N} / \mathrm{A}$ & $0.5^{e}$ \\
\hline Wavelength $(\mathrm{nm})$ & 543 & 543 & 543 \\
\hline No. of runs averaged for each subject & 4 & 4 & 4 \\
\hline Mydriasis & $\begin{array}{l}1 \text { Drop } \\
\text { cyclopentolate } \\
1 \%\end{array}$ & $\begin{array}{l}\text { No Mydriasis required } \\
1 \text { Drop cyclopentolate } \\
1 \% \text { in these } \\
\text { measurements }\end{array}$ & $\begin{array}{l}1 \text { Drop } \\
\text { cyclopentolate } \\
1 \%\end{array}$ \\
\hline Light level required & $\sim 10 \mu \mathrm{W}$ & $\sim 0.1 \mu \mathrm{W}$ (Photopic) $)^{f}$ & $\sim 30 \mu \mathrm{W}$ \\
\hline Duration of the measurement & $\sim 5 \mathrm{~s}$ & $\sim 3$ or $4 \mathrm{~min}$ & $\sim 2 \mathrm{~s}$ \\
\hline Area available for each aerial image (pixels) & $64 \times 64$ & $\mathrm{~N} / \mathrm{A}$ & $15 \times 15$ \\
\hline Zernike terms fitted & 35 & 35 & 35 \\
\hline
\end{tabular}

\footnotetext{
a Abbreviations: LRT, Laser Ray Tracing; SRR, Spatially Resolved Refractometer; H-S, Hartmann-Shack Wavefront Sensor.

${ }^{b}$ Specifications of the microlens array: focal length $\mathrm{f}^{\prime}=50 \mathrm{~mm}$; clear aperture $=500 \mu \mathrm{m}$; lenslet interdistance $=600 \mu \mathrm{m}$; hexagonal arrangement.

${ }^{c}$ The effective pupil diameter is the distance from the pupil center required for all the light from each aperture to enter (or exit) the eye. For each technique, the wave aberration was computed for the corresponding effective pupil size, and then the Zernike coefficients are recalculated for the smallest pupil $(6.6 \mathrm{~mm})$ to allow direct comparison.

${ }^{d}$ Artificial pupil located in a plane conjugate to the eye's pupil.

e Equal to the microlens diameter.

${ }^{f}$ Retinal illuminance is about $10 \mathrm{td}$ when considering both the measurement beam and the extended background field.
}

used the SRR built at the Schepens Eye Research Institute (Boston, $\mathrm{MA}){ }^{2}$ whereas the H-S sensor and the LRT technique were implemented at the Institute of Optics (Madrid, Spain). ${ }^{14}$ The experimental conditions were kept as similar as possible, taking into account the inherent differences among methods.

Two subjects, one male aged 41 years (RN) and one female aged 25 years (EM), participated in the study, both using their right eye. In all three types of measurements, defocus was left uncorrected $(-1.5 \mathrm{D}$ for RN and $-0.5 \mathrm{D}$ for EM). Measurements were carried out over a 2-year period. For each instrument, sessions consisted of four runs of measurements from which the set of average Zernike coefficients (for comparison between techniques) and the corresponding standard deviation (for variability estimation within each technique) were computed.

\section{RESULTS}

The resulting Zernike coefficients are shown in Fig. 2 for subjects RN (a) and EM (b). Solid triangles represent values for SRR, open circles represent LRT, and solid squares represent H-S. Each symbol is the average value from four runs. The error bars (representing the standard deviation of each technique) have not been depicted for the sake of clarity because they are all the same size as the symbol or smaller. The ordering for the Zernike polynomials corresponds to that recommended by the Optical Society of America Standardization Committee. ${ }^{22}$ We assessed the variability within a technique by computing the standard deviation of each Zernike coefficient across runs in a session and then taking the average across coefficients. The standard deviation within each technique was compared with the average standard deviation across techniques. For this purpose, we averaged the set of Zernike Coefficients obtained with LRT, SRR, and H-S, computed the standard deviation of each individual coefficient, and then averaged these values across the coefficients. Averaging was done either for coefficients 3 to 35 (orders 2-7) or for coefficients 3 to 14 (orders 2-4) to analyze possible changes across orders.

There was a close match among the three techniques: For RN (RMS wavefront error $0.5 \mu \mathrm{m}$ ) the average standard deviation across data (Zernike coefficients) from an individual technique was $0.071 \mu \mathrm{m}(0.066 \mu \mathrm{m}$ for H-S, 0.063 for LRT, and 0.084 for SRR), whereas the average standard deviation across the three techniques was $0.09 \mu \mathrm{m}$. For subject EM (RMS wavefront error 0.9 $\mu \mathrm{m})$, the average standard deviation of data from individual techniques was $0.065 \mu \mathrm{m}$, whereas the average standard deviation across the three techniques was again $0.09 \mu \mathrm{m}$. The main contribution to the slight mismatch across techniques was due to the low-order aberrations, which present the largest aberration values. 


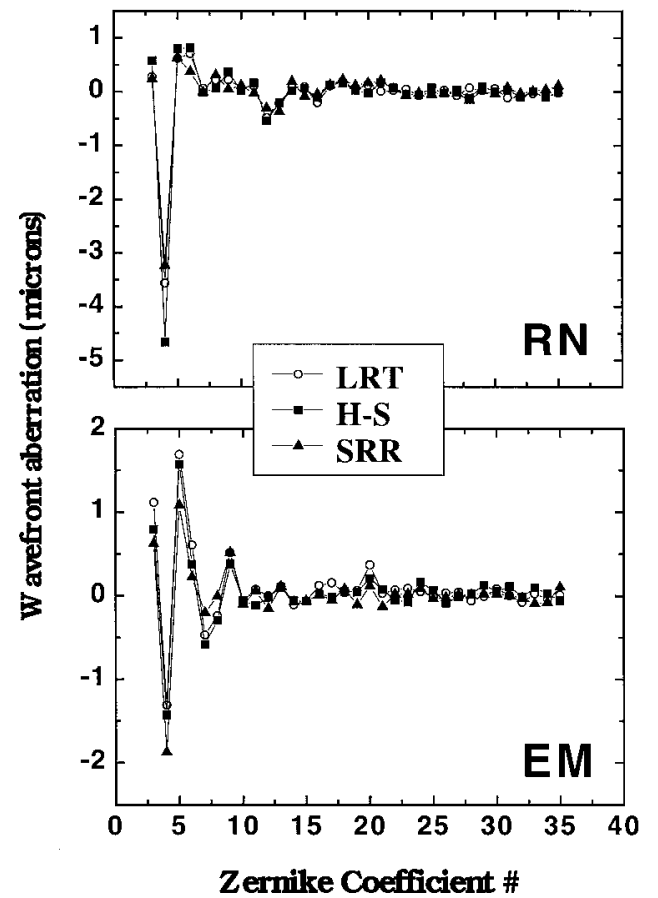

FIGURE 2.

Zernike coefficients for the three techniques for subjects RN (upper) and EM (lower). Triangles represent SRR, open circles represent LRT, and solid squares represent $\mathrm{H}-\mathrm{S}$. Each symbol is the average across four runs. Errors bars (not shown) are the same size or smaller than the symbols in all cases.

Considering only orders 2 to 4 (coefficients 3 to 14 ), the average standard deviation across techniques was $0.16 \mu \mathrm{m}$, and the standard deviation within a technique was $0.09 \mu \mathrm{m}$ for RN; the standard deviations were $0.14 \mu \mathrm{m}$ and $0.08 \mu \mathrm{m}$, respectively, for EM. The main contribution to the larger intertechnique standard deviation was due to the defocus term, which is the one most likely to change across sessions due to the variation of the accommodative state reached by the subject after instillation of the mydriatic agent.

Fig. 3 shows contour plots of the wave aberration for RN for each of the three methods. Tilt terms $\left(Z_{1}\right.$ and $\left.Z_{2}\right)$ and defocus $\left(Z_{4}\right)$ have been cancelled in these plots to enhance the high-order fea-

\section{Subject RN}

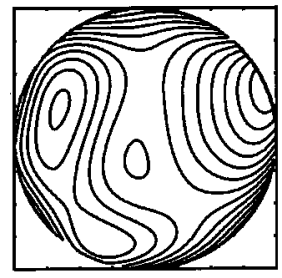

H-S

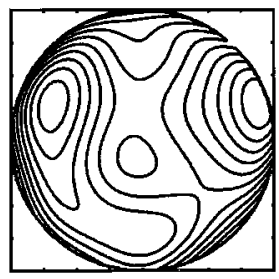

LRT

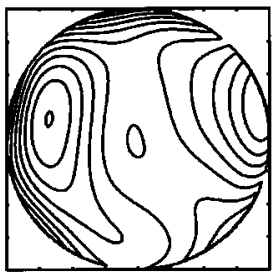

SRR

\section{FIGURE 3.}

Contour plots of the wave aberration for the right eye of subject RN for $\mathrm{H}-\mathrm{S}$ (left), LRT (center), and SRR (right) measurements. Left represents temporal side, and right represents nasal side. Pupil coordinates range from -3.25 $\mathrm{mm}$ to $3.25 \mathrm{~mm}$. Step between adjacent contour lines is $0.5 \mu \mathrm{m}$. Tilt terms $\left(Z_{1}\right.$ and $\left.Z_{2}\right)$ and defocus $\left(Z_{4}\right)$ have been cancelled. tures of the wave aberration, showing also the equivalence of the three methods for the measurement of optical aberrations in normal subjects. Nevertheless, given their different nature, each one can present advantages or limitations depending on the specific application.

\section{DISCUSSION}

The results of this study and the main differences among the three techniques are summarized below. We emphasize both issues that are inherent to the nature of each method and issues related to our particular implementation.

First, for the two normal subjects presented here, the three methods are equivalent. However, some differences may arise when measuring non-normal eyes. For example, for eyes with considerable amounts of wave aberrations, LRT and SSR may be more robust than H-S because of their sequential nature. That is, because they are sequential, they do not suffer from indexing problems, ${ }^{8}$ which may appear in H-S if the grid of image spots is too distorted. Alternatively, the computation of the centroid may be affected by speckle noise and the limited number of pixels/spot in $\mathrm{H}-\mathrm{S}^{14}$; when the spots are too distorted (for highly aberrated eyes) and the boundary between adjacent spots is not clear, the centroid computations become difficult, even for a diffraction-limited first pass. ${ }^{14}$ This may limit its range of application, especially in some clinically interesting cases. Alternative light sources (such as superluminescent diodes) have been suggested, to overcome the speckle noise problem in H-S. This limitation is reduced in LRT, in which the entire CCD is used for each spot. In SRR, the only limitation is the ability of the subject to align the image spot and the dynamic range of the gimbaled mirror.

Second, measurements with LRT and H-S rely on a good retinal reflection, which might be a problem in abnormal retinas. ${ }^{23}$ This is not a hindrance for SRR; however, SRR requires proper light capture by the photoreceptors and good fixation. These requirements could be a disadvantage for some classes of patients.

Third, the sequential nature of LRT and SRR limits their application for real-time dynamic measurements. H-S is the fastest technique ( 1 or $2 \mathrm{~s}$ ), and speckle removal techniques can be applied to take faster real-time dynamic recordings. ${ }^{24}$ In the present configuration, the duration of each exposure in LRT can be reduced to $-50 \mathrm{~ms}$ without being limited by speckle noise, ${ }^{14}$ the main constraint in duration of a measurement being the speed of the imaging board. This implementation requires 5 s for 37 rays. An SRR run takes 3 to 4 min due to its psychophysical nature. Although eye movements, fluctuations of accommodation, etc., may be thought to be more important problems for the slowest techniques, we did not find a trend for increased variability with a particular technique: for RN, SRR was the noisiest $(0.083 \mu \mathrm{m})$; for EM, H-S was the noisiest $(0.09 \mu \mathrm{m})$.

The following two aspects are related to the particular implementation of the technique used in the study and thus could be overcome in future versions of the system. First, all three systems operate with visible light (although some versions of H-S with infrared light have already been used by other groups ${ }^{24}$ ), and as a result, pupil dilation is required for H-S and LRT to avoid reflex pupil constriction. SRR uses light levels sufficiently low to operate with a natural pupil for most subjects (although for this work, a 
midriatic was used for comparison with the other methods. In this sense, it can therefore be more comfortable for the subject.

Second, H-S uses a fixed monolithic microlens array; the sampling configuration cannot be modified unless different microlens arrays are used. In the SRR version used in this work, the sampling pattern provided by the wheel was also fixed, although programmable masks are possible. ${ }^{25}$ In LRT, the laser scanner can be flexibly programmed to change the shape of the sampling pattern (rectangular, hexagonal, and polar). This allows the sampling pattern to be adapted to individual cases (i.e., normal vs. refractive surgery patients) or even the use of a nonhomogeneous sampling pattern. ${ }^{26}$ Even if the sampling step size can be changed easily, the sample size (given by the beam waist size) remains unchanged, which affects the "filling factor" (fraction of the pupil covered by the sampling pattern).

In summary, we have demonstrated experimentally the equivalence of three different techniques for measuring ocular aberrations in normal human eyes: the laser ray tracing, the spatially resolved refractometer and the Hartmann-Shack wavefront sensor. The particular features of each technique (see table) make one or the other more convenient depending on the specific problem or application.

\section{ACKNOWLEDGMENTS}

We thank Mike Hutley for providing the microlens array used in the experiments. This research was supported by grant TIC98 to 0925-C02 to 01 from the Comision Interministerial de Ciencia y Tecnologia of Spain, grant EY04395 from the National Eye Institute, National Institutes of Health, and by the Human Frontier Science Program LT-542/97.

Received August 21, 2000; revision received December 7, 2000.

\section{REFERENCES}

1. Walsh G, Charman WN, Howland HC. Objective technique for the determination of monochromatic aberrations of the human eye. $\mathrm{J}$ Opt Soc Am (A) 1984;1:987-92.

2. He JC, Marcos S, Webb RH, Burns SA. Measurement of the wavefront aberration of the eye by a fast psychophysical procedure. J Opt Soc Am (A) Opt Image Sci Vis 1998;15:2449-56.

3. Liang J, Grimm B, Goelz S, Bille JF. Objective measurement of wave aberrations of the human eye with the use of a Hartmann-Shack wave-front sensor. J Opt Soc Am (A) 1994;11:1949-57.

4. Navarro R, Losada MA. Aberrations and relative efficiency of light pencils in the living human eye. Optom Vis Sci 1997;74:540-7.

5. Liang J, Williams DR. Aberrations and retinal image quality of the normal human eye. J Opt Soc Am (A) 1997;14:2873-83.

6. Iglesias I, Berrio E, Artal P. Estimates of the ocular wave aberration from pairs of double-pass retinal images. J Opt Soc Am (A) 1998;15: 2466-76.

7. Mierdel P, Krinke HE, Wiegand W, Kaemmerer M, Seiler T. Measuring device for determining monochromatic aberration of the human eye. Ophthalmologe 1997;94:441-5.

8. Thibos LN, Hong X. Clinical applications of the Shack-Hartmann aberrometer. Optom Vis Sci 1999;76:817-25.

9. Oshika T, Klyce SD, Applegate RA, Howland HC, El Danasoury MA. Comparison of corneal wavefront aberrations after photorefrac- tive keratectomy and laser in situ keratomileusis. Am J Ophthalmol 1999;127:1-7.

10. Liang J, Williams DR, Miller DT. Supernormal vision and highresolution retinal imaging through adaptive optics. J Opt Soc Am (A) 1997;14:2884-92.

11. Zhu L, Sun P, Bartsch D, Freeman WR, Fainman Y. Adaptive control of a micromachined continous-membrane deformable mirror for aberration compensation. Appl Opt 1999;38:168-76.

12. Navarro R, Moreno-Barriuso E, Bará S, Mancebo T. Phase-plates for wave-aberration compensation in the human eye. Opt Lett 2000;25: 236-8.

13. Salmon TO, Thibos LN, Bradley A. Comparison of the eye's wavefront aberration measured psychophysically and with the ShackHartmann wave-front sensor. J Opt Soc Am (A) 1998;15:2457-65.

14. Moreno-Barriuso E, Navarro R. Laser Ray Tracing versus HartmannShack sensor for measuring optical aberrations in the human eye. J Opt Soc Am (A) Opt Image Sci Vis 2000;17:974-85.

15. Thibos L, Hong X. Comparison of monochromatic aberrations of the human eye measured with the Howland crossed-cylinder aberroscope and the Shack-Hartmann aberrometer. In: Optical Society of America Annual Meeting, Technical Digest Series,1999. Washington, DC: Optical Society of America, 1999:55.

16. Webb RH, Penney CM, Thompson KP. Measurement of ocular wavefront distortion with a spatially resolved refractometer. Appl Opt 1992;31:3678-86.

17. Smirnov MS. Measurement of the wave aberration of the human eye. 1961:776-95.

18. Born M, Wolf E. Principles of Optics: Electromagnetic theory of Propagation, Intererence and Diffraction of Light, 6th ed. Oxford: Pergamon Press, 1993.

19. Malacara D. Optical Shop Testing, 2nd ed. New York: Wiley, 1992.

20. Southwell WH. Wave-front estimation from wave-front slope measurements. J Opt Soc Am (A) 1980;70:998-1006.

21. Navarro R, Losada MA. Phase transfer and point-spread function of the human eye determined by a new asymmetric double-pass method. J Opt Soc Am (A) 1995;12:2385-92.

22. Thibos LN, Applegate RA, Schwiegerling JT, Webb R, Burns S, VSIA Standards Taskforce Members. Report from the VSIA Taskforce on standards for the reporting of the optical aberrations of the eye. In: Vision Science and its Applications, 2000. Technical Digest Series. Washington, DC: Optical Society of America, 2000: 143-5.

23. Elsner AE, Burns SA, Weiter JJ, Delori FC. Infrared imaging of sub-retinal structures in the human ocular fundus. Vision Res 1996; 36:191-205.

24. Hofer HJ, Porter J, Williams DR. Dynamic measurement of the wave aberration of the human eye. Invest Ophthalmol Vis Sci 1998;39: S203.

25. Webb RH, Burns SA, Penney M. Coaxial spatially resolved refractometer. US Patent 6,000,800, 1999.

26. Rios S, Acosta E, Bara S. Hartmann sensing with Albrecht grids. Opt Comm 1997;133:443-53.

Esther Moreno

Instituto de Optica "Daza de Valdés" (C.S.I.C.)

Serrano 121

28006 Madrid, Spain

e-mail:e.moreno@io.cfmac.csic.es 